\title{
Benchmarking of measurement and simulation of transverse rms-emittance growth
}

\author{
L. Groening, W. Barth, W. Bayer, G. Clemente, L. Dahl, P. Forck, P. Gerhard, I. Hofmann, G. Riehl, and S. Yaramyshev \\ Gesellschaft für Schwerionenforschung, Planckstrasse 1, D-64291 Darmstadt, Germany \\ D. Jeon \\ Oak Ridge National Laboratory, Oak Ridge, Tennessee 37830, USA \\ D. Uriot \\ CEA IRFU, Service des Accélérateurs, de Cryogénie et de Magnétisme, F-91191 Gif-sur-Yvette, France
}

(Received 17 December 2007; published 18 September 2008)

\begin{abstract}
Transverse emittance growth along the Alvarez drift tube linac (DTL) section is a major concern with respect to the preservation of beam quality of high current beams at the GSI UNILAC. In order to define measures to reduce this growth, appropriate tools to simulate the beam dynamics are indispensable. This paper is about the benchmarking of three beam dynamics simulation codes, i.e. DYNAMION, PARMILA, and PARTRAN against systematic measurements of beam emittances for different transverse phase advances along the DTL. Special emphasis is put on the modeling of the initial distribution for the simulations. The concept of rms equivalence is expanded from full intensity to fractions of less than $100 \%$ of the beam. The experimental setup, data reduction, preparation of the simulations, and the evaluation of the simulations are described. In the experiments and in the simulations, a minimum of the rms-emittance growth was observed at zero current phase advances of about $60^{\circ}$. In general, good agreement was found between simulations and experiment for the mean values of horizontal and vertical emittances at the DTL exit.
\end{abstract}

DOI: 10.1103/PhysRevSTAB.11.094201

PACS numbers: 29.27.Bd, 41.75.-i, 41.85.-p

\section{INTRODUCTION}

At GSI currently the new Facility for Antiproton and Ion Research (FAIR) is under design [1]. It comprises two new superconducting synchrotrons and five storage rings to provide radioactive beams and exotic nuclei. The existing GSI facility will serve as an injector chain for FAIR and hence must deliver the primary beam intensities. It comprises the UNIversal Linear ACcelerator (UNILAC), the heavy ion synchrotron SIS, and an experimental storage ring.

The UNILAC [2] can accelerate ions from protons to uranium (Fig. 1). Beams from three different ion sources can be delivered to various experiments or to the SIS in pulse-switching mode. The original 1960s design did not foresee high intensity operation where space charge forces need to be considered in the beam dynamics layout. However, the UNILAC underwent several upgrades [2] and since 1999 many experiments were served successfully with high intensity beams.

With the upcoming FAIR project, the demands on high intensity beams to the UNILAC increased. Intense beams are provided by MEVVA, MUCIS, or CHORDIS sources at low charge states with the energy of $2.2 \mathrm{keV} / \mathrm{u}$. An radio frequency quadrupole (RFQ) followed by two IH-cavities [Hoch-Strom-Injektor (HSI) section] accelerates the ions to $1.4 \mathrm{MeV} / \mathrm{u}$ using an rf frequency of $36 \mathrm{MHz}$. A subsequent gas stripper increases the average charge state of the ion beam. Final acceleration to $11.4 \mathrm{MeV} / \mathrm{u}$ is done in the Alvarez drift tube linac (DTL) section operated at $108 \mathrm{MHz}$. The increase of rf frequency by a factor of 3 requires a dedicated matching section preceding the DTL as shown in Fig. 2. It comprises a $36 \mathrm{MHz}$ buncher for longitudinal bunch compression, a $108 \mathrm{MHz}$ buncher for final bunch rotation, a quadrupole doublet for transverse compression, and a quadrupole triplet for final transverse beam matching.

The Alvarez DTL consists of five independent rf tanks accelerating to $3.6,4.8,5.9,8.6$, and $11.4 \mathrm{MeV} / \mathrm{u}$, respectively. Transverse beam focusing is performed by quadrupoles in the F-D-D-F mode. Each drift tube houses one quadrupole. The periodicity of the lattice is interrupted by four intertank sections, where D-F-D focusing is applied. Acceleration is done $-30^{\circ}$ from crest in the first three tanks and $-25^{\circ}$ from crest in the last two tanks. Different beam energies behind the DTL are available by switching off the acceleration in the last tanks. However, the production mode for FAIR foresees full acceleration to 11.4 $\mathrm{MeV} / \mathrm{u}$ with the design beam parameters as summarized in Table I. Because of its high rigidity, the uranium ion imposes the strongest requirements to the UNILAC. Table I therefore refers to operation with uranium. The DTL is followed by a section of ten single gap resonators for energy variation behind the DTL. They were not used for the experiments described here. 


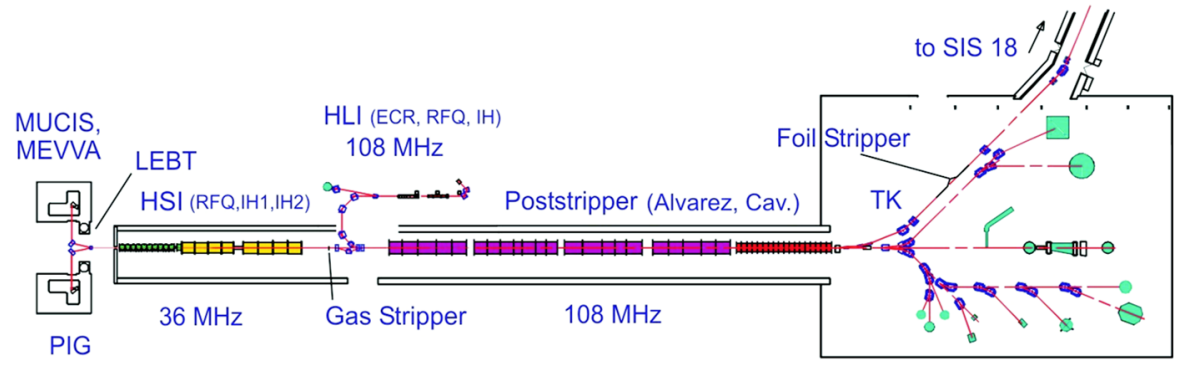

FIG. 1. (Color) The UNIversal Linear ACcelerator UNILAC at GSI.

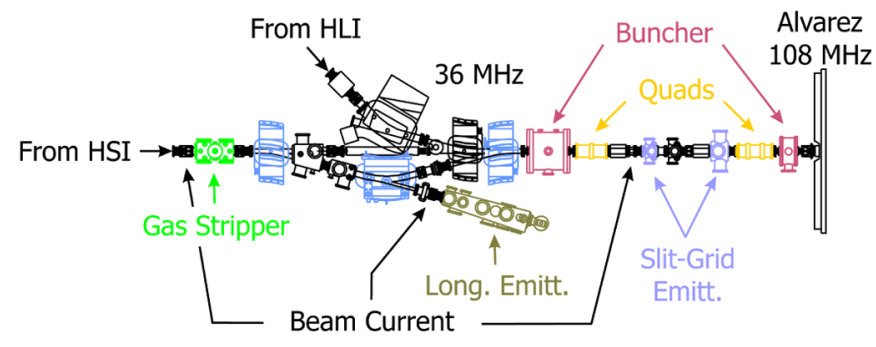

FIG. 2. (Color) The stripper section including the matching section to the Alvarez DTL.

TABLE I. Design beam parameters of the UNILAC for uranium as required by FAIR. The term $\beta \gamma \epsilon_{\perp}$ refers to the normalized, total transverse emittance.

\begin{tabular}{lccc}
\hline \hline & $\begin{array}{c}\text { RFQ } \\
\text { entrance }\end{array}$ & $\begin{array}{c}\text { DTL } \\
\text { entrance }\end{array}$ & $\begin{array}{c}\text { SIS } \\
\text { injection }\end{array}$ \\
\hline Ion species & ${ }^{238} \mathrm{U}^{4+}$ & ${ }^{238} \mathrm{U}^{28+}$ & ${ }^{238} \mathrm{U}^{28+}$ \\
Pulse current $[\mathrm{mA}]$ & 18 & 15 & 15 \\
Energy $[\mathrm{MeV} / \mathrm{u}]$ & 0.002 & 1.39 & 11.4 \\
Momentum spread (relative) & $\cdots$ & $\pm 5 \times 10^{-3}$ & $\pm 1 \times 10^{-3}$ \\
$\beta \gamma \epsilon_{\perp}[\mathrm{mm}$ mrad] & 0.30 & 0.75 & 1.1 \\
\hline \hline
\end{tabular}

Injection into the synchrotron SIS is done using horizontal multiturn injection. This scheme imposes an upper limit for the horizontal emittance at the injection point thus reducing the budget for possible emittance growth during beam acceleration and transport. Previous beam experiments revealed transverse growth rates of up to a factor of 5 just along the DTL section at high currents. In order to reduce this growth, its course has to be investigated experimentally accompanied by beam dynamics simulations.

However, any hardware modification aimed at reducing the growth should be checked by simulations before being implemented into the real machine. This step is based on the reliability of the respective simulation code, in other words, on the knowledge of the limits of the applied code. Additionally, benchmarking of high intensity linac simulation codes would be desirable to enhance credibility of future high power linac design in Europe and elsewhere. Therefore, a benchmark activity had been integrated into the Joint Research Activity CARE (Coordinated Accelerator Research in Europe) [3] supported by the European Commission. It aims at the simulation of systematic beam emittance measurements performed at the DTL entrance and exit, respectively.

For the simulations three different codes were used: DYNAMION [4], PARMILA (V 2.32) [5], and PARTRAN [6]. The first code uses 3D-particle-particle interaction of a few thousand particles and the other codes use PICNIC3D Poisson solvers with some $10^{5}$ particles. The rf fields are calculated from the drift tube geometry for all codes. DYNAMION solves the Laplace equation to generate the electric field on a grid followed by particle tracking. PARMILA and PARTRAN use tables of time transit factors generated by SUPERFISH to apply nonlinear kicks to the particles. Table II summarizes the codes properties.

A previous benchmarking study on halo formation was performed at Los Alamos. It compared measured transverse profiles of a high intensity proton beam with simulated profiles along a transport channel without acceleration $[7,8]$. Benchmarking among eight different simulation codes was performed prior to the UNILAC experiments $[9,10]$. These studies strongly triggered and supported code improvement and bug fixing.

Measurements with ${ }^{238} \mathrm{U}^{28+}$ showed that the transverse emittance growth depends on the DTL quadrupole settings, i.e., on the phase advance [11]. These findings called for a more detailed experimental investigation of the dependence of emittance growth on transverse focusing strength.

TABLE II. Summary of the properties of the simulation codes.

\begin{tabular}{lccccc}
\hline \hline & Solver & Boundaries & Number of particles & CPU time & Rf-Gap \\
\hline DYNAMION & 3D-particle-particle & Open & $4.3 \times 10^{3}$ & $20 \mathrm{~h}$ & Tracking \\
PARMILA & PICNIC-3D & Open & $2 \times 10^{5}$ & $30 \mathrm{~min}$ & Nonlinear kicks \\
PARTRAN & PICNIC-3D & Open & $2 \times 10^{5}$ & $30 \mathrm{~min}$ & Nonlinear kicks \\
\hline \hline
\end{tabular}


Because of the large mass over charge ratio of ${ }^{238} \mathrm{U}^{28+}$, the maximum zero current phase advance $\sigma_{o}$ that can be applied along the DTL is $54^{\circ}$. Additionally, the maximum current achieved so far with ${ }^{238} \mathrm{U}^{28+}$ is $5.5 \mathrm{~mA}$ at the DTL entrance thus not providing the space charge conditions at the design current of $15 \mathrm{~mA}$. The two limitations could be removed by using a ${ }^{40} \mathrm{Ar}^{10+}$ beam which can be delivered with currents of more than $10 \mathrm{~mA}$ at the DTL entrance. The maximum $\sigma_{o}$ applicable to ${ }^{40} \mathrm{Ar}^{10+}$ exceeds $180^{\circ}$. Space charge forces scale as $I \cdot q / A$, where $I$ is the pulse beam current, $q$ is the ions charge state, and $A$ is its mass number. Hence a $7.1 \mathrm{~mA}$ beam of ${ }^{40} \mathrm{Ar}^{10+}$ is space charge equivalent to a $15 \mathrm{~mA}$ beam of ${ }^{238} \mathrm{U}^{28+}$.

\section{EXPERIMENT SETUP AND PROCEDURE}

Figure 3 presents the schematic setup of the experiments. A high current beam of ${ }^{40} \mathrm{Ar}^{1+}$ is delivered from the HSI and passed through the gas stripper. A system composed of three dipoles and horizontal scrapers selects the desired charge state of $10+$.

One beam current transformer is placed in front of the DTL between the quadrupole doublet and triplet. Additional transformers are located after tanks A1, A2b, $\mathrm{A} 3$, and A4, respectively. They are used to measure the beam transmission along the DTL. Horizontal and vertical profile grids are located in front of the first tank and behind each of the Alvarez tanks. The ones in front of and behind the DTL are equipped with horizontal and vertical slits and are used as transverse emittance measurement units. Each measurement takes about five minutes per plane with a resolution of $0.8 \mathrm{~mm}$ in space and $0.5 \mathrm{mrad}$ in angle. During one measurement several $10^{5}$ bunches impact on the measurement device, thus the final result represents the average emittance of a bunch train. The total accuracy of each rms-emittance measurement including its evaluation is estimated to be $10 \%$.

A setup to measure the longitudinal rms-bunch length is available in front of the DTL [12]. It measures the time of impact of a single ion on a foil. This time is related to a $36 \mathrm{MHz}$ master oscillator. The resolution is $0.3^{\circ}$ (36 MHz).
One measurement of rms bunch length takes about 20 minutes.

A second setup is installed in a dispersive section behind the DTL. It comprises one circular iris, a dispersion-rising bend, a vertically installed buncher operated at $108 \mathrm{MHz}$, a focusing doublet, and a fluorescence screen. Because of dispersion at the screen, the particles energy deviation is transformed linearly into horizontal displacement. The vertically deflecting buncher transforms the rf phase of a particle into vertical deflection being proportional to the $\mathrm{rf}$ phase (within $\approx \pm 30^{\circ}$ ). If the beam spot size at the screen behind the buncher is dominated by rf-phase spread and by energy spread, the beam image can be regarded as an online image of the longitudinal phase space distribution. This is the case if the intrinsic beam size is strongly reduced by insertion of the circular iris in front of the dispersion-rising bend. The setup was used to detect eventual low-energy beam tails at the DTL exit. Its extension towards a tool for quantitative longitudinal emittance measurements is ongoing.

The experiments were performed with an intense ${ }^{40} \mathrm{Ar}^{1+}$ beam from the HSI at $1.4 \mathrm{MeV} / \mathrm{u}$. The gas stripper was set to obtain $7.1 \mathrm{~mA}$ of ${ }^{40} \mathrm{Ar}^{10+}$ in front of the DTL. Horizontal and vertical phase space distributions were measured in front of the DTL as presented in Fig. 4. Additionally, the longitudinal rms-bunch length was measured at the entrance to the DTL.

The DTL quadrupoles were set to different zero current transverse phase advances $\sigma_{o}$ ranging from $35^{\circ}$ to $90^{\circ}$ in steps of $5^{\circ}$. Because of the defocusing space charge forces, the phase advances in all three dimensions were depressed as listed in Table III. As mentioned in Sec. I, the DTL comprises five tanks separated by four intertank sections. Each section includes a triplet. The four triplets were set to match the periodic solutions of adjacent tanks for zero current.

After setting the desired $\sigma_{o}$ along the DTL, the quadrupoles and bunchers preceding the DTL were set to obtain full transmission through the DTL and to minimize lowenergy tails of the beam. For each value of $\sigma_{o}$ horizontal and vertical beam emittances were measured at the exit of

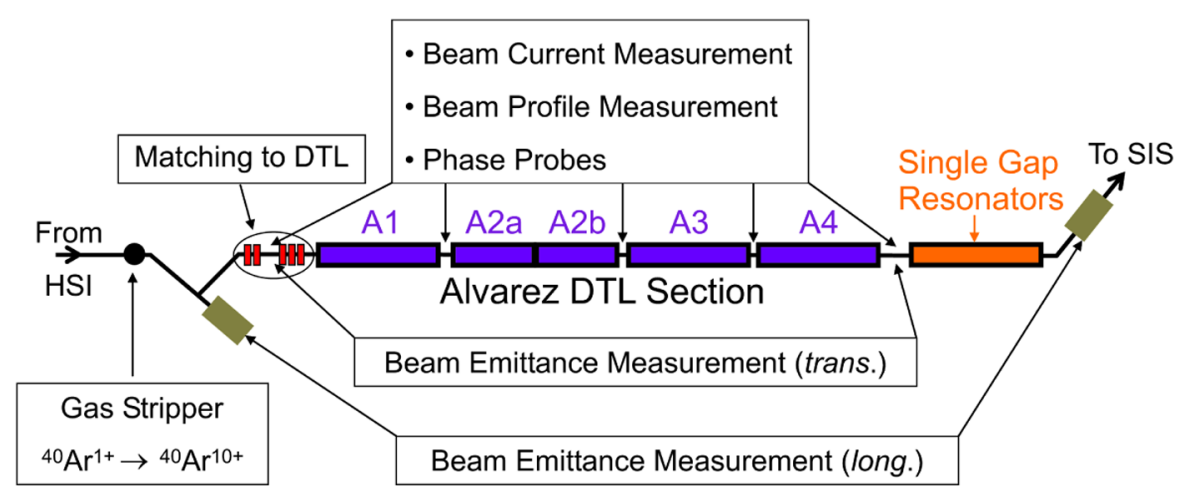

FIG. 3. (Color) Schematic setup of the experiments. 

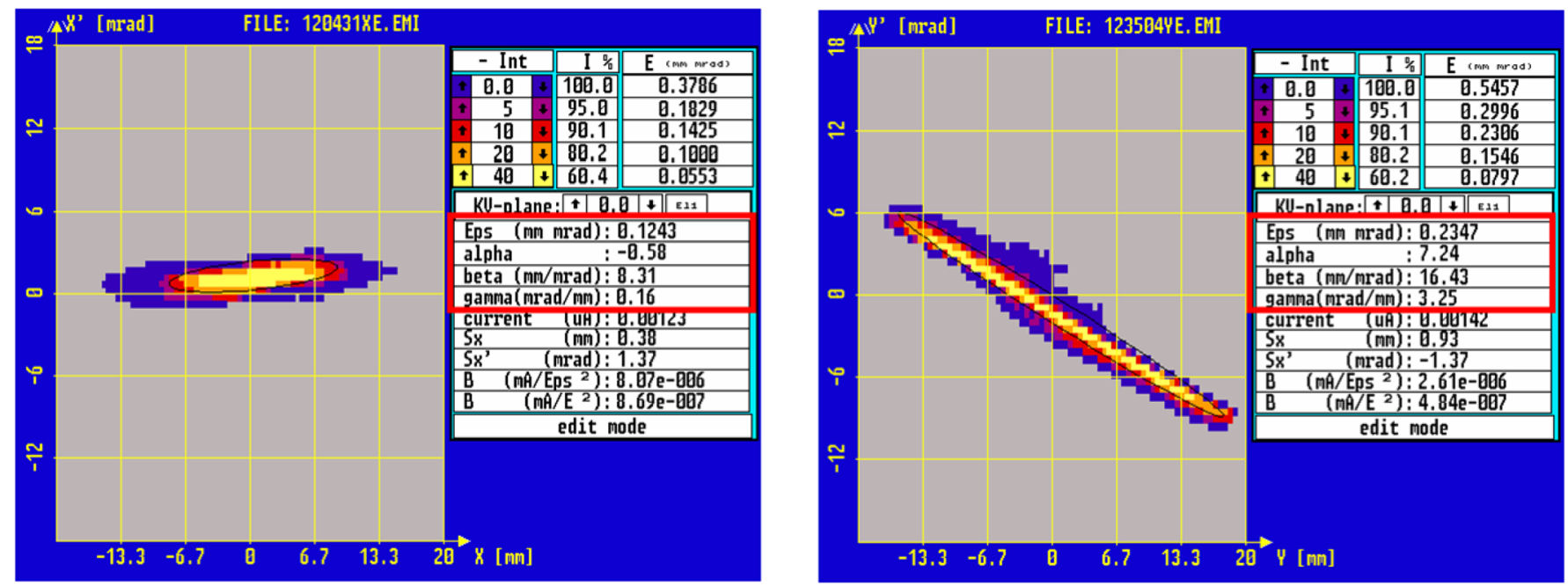

FIG. 4. (Color) Horizontal (left) and vertical (right) phase space distribution measured in front of the DTL for a $7.1 \mathrm{~mA}$ beam of ${ }^{40} \mathrm{Ar}^{10+}$. Normalized rms-Twiss parameters corresponding to $100 \%$ of the total intensity are highlighted in red.

TABLE III. Horizontal, vertical, and longitudinal phase advances along the DTL in case of zero current (left) and of $7.1 \mathrm{~mA}$ of ${ }^{40} \mathrm{Ar}^{10+}$ (right) for an rms-equivalent KV-beam. The corresponding normalized $100 \% \mathrm{rms}$ emittances are $0.12 / 0.23 \mathrm{~mm} \mathrm{mrad}$ (horizontal/vertical) and $66 \mathrm{deg} \mathrm{mrad}$ (referring to $108 \mathrm{MHz}$ and to relative momentum deviation).

\begin{tabular}{lcc|ccc}
\hline \hline Horizontal & $\begin{array}{c}\sigma_{o}[\mathrm{deg}] \\
\text { Vertical }\end{array}$ & Longitudinal & Horizontal & $\begin{array}{c}\sigma[\mathrm{deg}] \\
\text { Vertical }\end{array}$ & Longitudinal \\
\hline 35 & 35 & 43 & 18 & 22 & 39 \\
40 & 40 & 43 & 22 & 27 & 39 \\
45 & 45 & 43 & 26 & 31 & 39 \\
50 & 50 & 43 & 31 & 36 & 38 \\
55 & 55 & 43 & 35 & 41 & 38 \\
60 & 60 & 43 & 40 & 45 & 38 \\
65 & 65 & 43 & 44 & 50 & 37 \\
70 & 70 & 43 & 49 & 55 & 37 \\
75 & 75 & 43 & 54 & 60 & 37 \\
80 & 80 & 43 & 58 & 64 & 37 \\
85 & 85 & 43 & 63 & 69 & 37 \\
90 & 90 & 43 & 68 & 74 & 36 \\
\hline \hline
\end{tabular}

the DTL. In order to check the reproducibility of the measurements, seven settings of $\sigma_{o}$ were measured twice. The observed differences between two measurements using the same settings were just a few percent in rms emittances. After the full $\sigma_{o}$ scan, the initial transverse emittances in front of the DTL were remeasured as well as the initial rms bunch length. The measurements confirmed that the initial beam conditions did not change during the experiment.

\section{EXPERIMENTAL DATA REDUCTION}

Each emittance measurement delivers a twodimensional matrix of discrete slit positions and discrete angles. To each element of the matrix, a value is appointed that corresponds to the density of phase space population at this phase space coordinate (pixel). The data are displayed by the measurement and evaluation program PROEMI [13]. For the measured distribution, the $100 \%$ rms emittance is calculated considering the statistical weight of each pixel which is proportional to the pixel content (in the following the term emittance refers to the phase space area divided by $\pi)$.

Alternatively, fractional emittances can be extracted from the distribution as well. This is useful for distributions that are not homogeneous and where the Twiss parameters of a dense core differ considerably from those of the full distribution. In practical cases it is beneficial to focus on 
the "inner" $95 \%$ of the particles instead of putting huge efforts to achieve an optic that fits to the $100 \%$ Twiss parameters. The emittance containing a given percentage $p$ of the full distribution is extracted as follows: (i) The $\operatorname{sum} \sum_{100}$ of all pixel contents is calculated. (ii) The pixels are sorted by their content starting from the largest one. (iii) Starting from the largest content the sum of all pixel contents is built as long as this sum is less or equal to the percentage $p$ of $\sum_{100}$. (iv) Those pixels that contributed to the sum are considered for the rms evaluation. The content of the others is omitted.

\section{EVALUATION OF SIMULATION RESULTS}

In order to compare the results of measurements with simulations adequately, it must be assured that the simulated emittances are defined in the same way as their experimental pendants. This applies especially for the evaluation of fractional emittances from simulations. Each simulation delivers a set of six-dimensional particle coordinates. This ensemble is projected onto a pixel grid having the same characteristics as the slit/grid device used for the measurements. The grid is read by the measurement evaluation program PROEMI such that data reduction was done in the same way as for measured data (Sec. III). Figure 5 illustrates the transformation of format.

\section{PREPARATION OF INPUT FOR SIMULATIONS}

From the transverse emittances measured in front of the DTL (Fig. 4) normalized $100 \%$ rms emittances of 0.12 and $0.23 \mathrm{~mm}$ mrad were evaluated horizontally and vertically, respectively. Measuring the rms bunch length in front of the DTL a value of $25 \mathrm{~mm}$ was found corresponding to a phase spread of $20^{\circ}$ at $36 \mathrm{MHz}$.

The reconstruction of the initial phase space distribution was complicated by incomplete information on the longitudinal distribution and by the fact that the transverse data and the longitudinal data were measured at different positions as drawn in Fig. 6. In the following, the reconstruction of the initial distribution is done in two steps. First the 100\%-rms-Twiss parameters, i.e., emittances, beta functions, and alpha parameters, of the initial distribution are determined. In the second step the type of distribution is reconstructed.

\section{A. Initial rms-Twiss parameters}

The transverse measurements and the longitudinal measurements on the initial distribution done at different locations along the beam line had to be combined. This was done by the following procedure: (i) The measured DTL transmission is very sensitive to the setting of the bunchers. Changes of a few percent in voltages and phases lead to

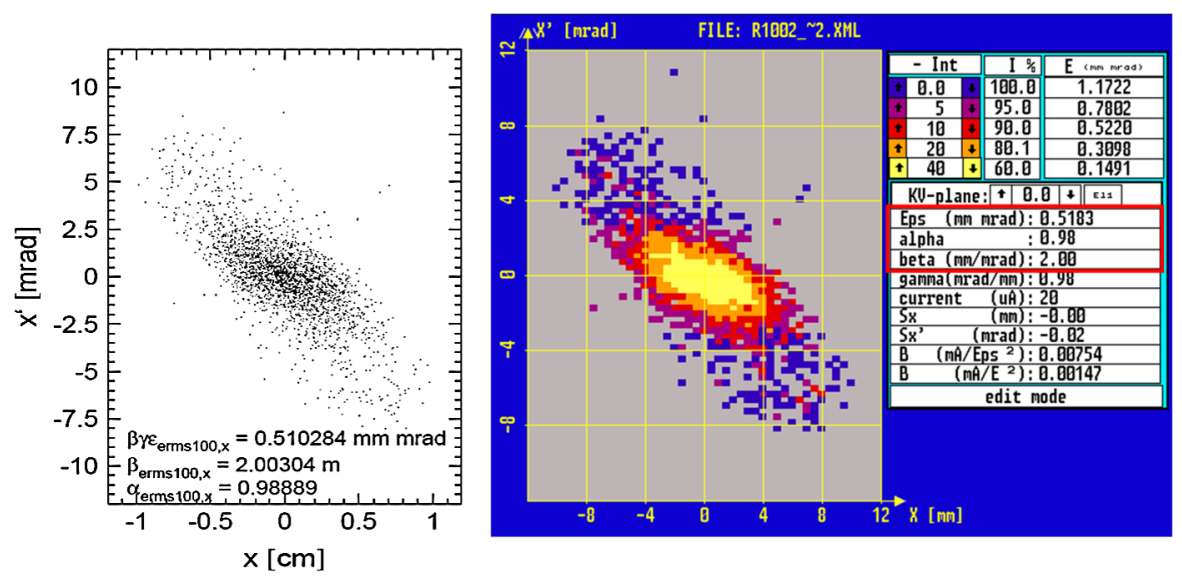

FIG. 5. (Color) Transformation of format of a distribution from DYNAMION simulation (left) to the format of measured data (right). Normalized $100 \%$-rms-Twiss parameters are highlighted in red.

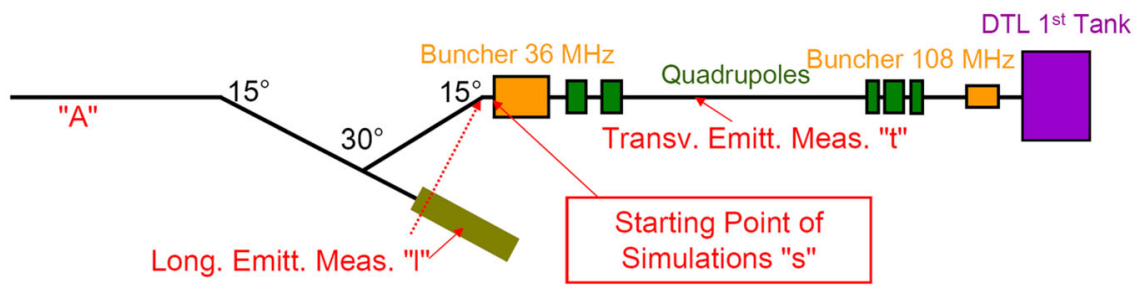

FIG. 6. (Color) Reference points used for reconstruction of the initial phase space distribution as needed for the simulations. 
measurable DTL transmission loss and to observable lowenergy tails. Full DTL transmission together with minimization of these tails therefore is a good indicator for longitudinally matched injection into the DTL. The settings during the experiments gave full transmission such that longitudinally matched injection can be assumed. (ii) The distance from the location " $\mathrm{A}$ " in the beam line to the location "s" where the simulations start is just $0.4 \mathrm{~m}$ longer with respect to the distance from " $\mathrm{A}$ " to the location "l" where the bunch length is measured. A virtual transport from "l" to " $\mathrm{s}$ " is introduced as a drift with space charge, i.e., neglecting dispersion. (iii) At location " $t$ ", the measured horizontal and vertical Twiss parameters are used to create rms-equivalent Kapchinsky-Vladimirsky (KV) distributions. The obtained transverse distributions are combined with a longitudinal KV distribution using guessed Twiss parameters. Any correlations between coordinates of different planes are assumed to be zero. For the transverse distributions the measured Twiss parameters are taken. (iv) The 6D-distribution constructed in "(iii)" is rms tracked backwards to location "s" plus additional $0.4 \mathrm{~m}$ [see "(ii)"]. The tracking includes the space charge forces of rms-equivalent KV distributions [14]. (v) The resulting rms bunch length is compared with the measured one at "l". In case of no agreement, the procedure must start again at "(iii)" using a different guess on the longitudinal Twiss parameters. (vi) In case of agreement, the corresponding Twiss parameters at "s" are used as input for a DTL matching routine (developed and tested after the campaign, see Sec. A). The setting for bunchers suggested by the routine is compared with the settings used during the experiments. In case of no agreement the procedure must start again at "(iii)". (vii) In case of agreement the rmsTwiss parameters of the initial distribution at location "s" are assumed to be reconstructed.

The initial longitudinal $100 \%$ rms emittance was reconstructed as $22 \mathrm{deg} \mathrm{mrad}$. The value refers to $36 \mathrm{MHz}$ and to the relative momentum deviation.

\section{B. Type of initial distribution}

The emittance growth strongly depends on the kind of distribution. Therefore the knowledge of the rms properties of the full distribution might be not sufficient for predictions on the growth. The amount of halo in the measured initial distribution should be included into the initial distribution used for the simulations. This amount can be estimated by evaluating the fractional rms emittances of the measurements in front of the DTL as described in Sec. III. Plotting the fractional rms emittance as a function of the fraction delivers a brilliance curve. In case of a homogeneous distribution, i.e., no halo, the curve is just a straight line. For distributions with a halo the curvature of the graph is increased. The brilliance curve corresponding to the horizontal emittance measurement in front of the DTL is presented in Fig. 7. The red curve indicates the

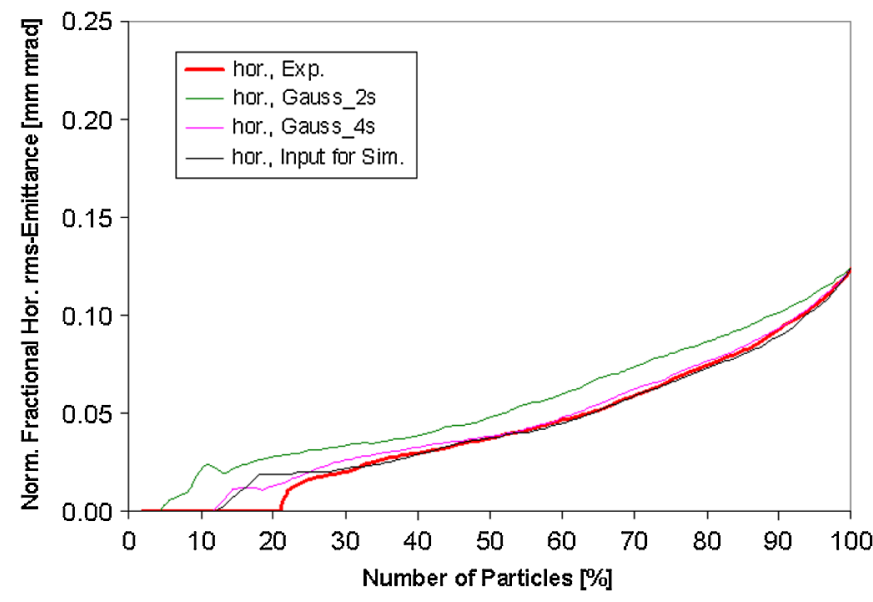

FIG. 7. (Color) Brilliance curves of the horizontal phase space distribution in front of the DTL. The curves were extracted from the measured distribution (red bold), a Gaussian cut at $2 \sigma$ (green), a Gaussian cut at $4 \sigma$ (pink), and from the distribution used for the simulations (black).

brilliance curve obtained from the measured data. The other lines indicate the curves for different distribution types. All lines must converge to the same value at the fraction of $100 \%$ but their shape might differ considerably for lower fractions. Since the curves are extracted from the emittance evaluation program based on phase space pixels and not on single particles, the curves might deliver zero emittance for very low fractions. This is just due to the fact that for very low fractions less than three pixels remain to be evaluated. The rms evaluation makes no sense in that case.

Figure 7 reveals that the horizontal brilliance curve of the initially measured distribution can be well reproduced by a Gaussian distribution cut at $4 \sigma$. Cutting at $2 \sigma$ instead

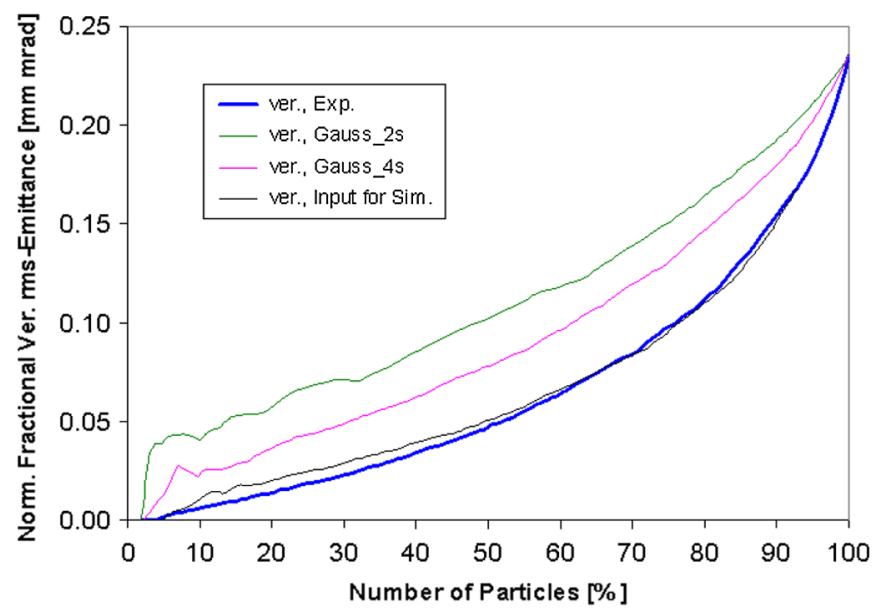

FIG. 8. (Color) Brilliance curves of the vertical phase space distribution in front of the DTL. The curves were extracted from the measured distribution (blue bold), a Gaussian cut at $2 \sigma$ (green), a Gaussian cut at $4 \sigma$ (pink), and from the distribution used for the simulations (black). 
would underestimate the amount of halo in the horizontal plane. Evaluating the vertical brilliance curve (Fig. 8) delivers an even stronger halo with respect to the horizontal projection of the initially measured distribution. Using a Gaussian cut at $4 \sigma$ still would underestimate the vertical halo significantly.

In order to reproduce the brilliance curves in both transverse projections an appropriate six-dimensional distribution function must be found. This was achieved by defining generalized coordinates $X, X^{\prime}, Y, Y^{\prime}, \Phi$, and $\delta P / P$ as well as an infinitesimal volume element in the six-dimensional phase space as

$$
d V=\Delta X \cdot \Delta X^{\prime} \cdot \Delta Y \cdot \Delta Y^{\prime} \cdot \Delta \Phi \cdot \Delta(\delta P / P) .
$$

The distribution function $f$ is

$$
\frac{d N}{d V}=f\left(X, X^{\prime}, Y, Y^{\prime}, \Phi, \delta P / P\right),
$$

with $d N$ being the number of particles within the volume element $d V$. After distributing the single particles according to $f$, the real particle coordinates are obtained from the generalized ones by applying virtual transport matrices to the distribution in each of the three planes. These matrices just contain the Twiss parameters of the distribution in generalized coordinates and in real coordinates. The later
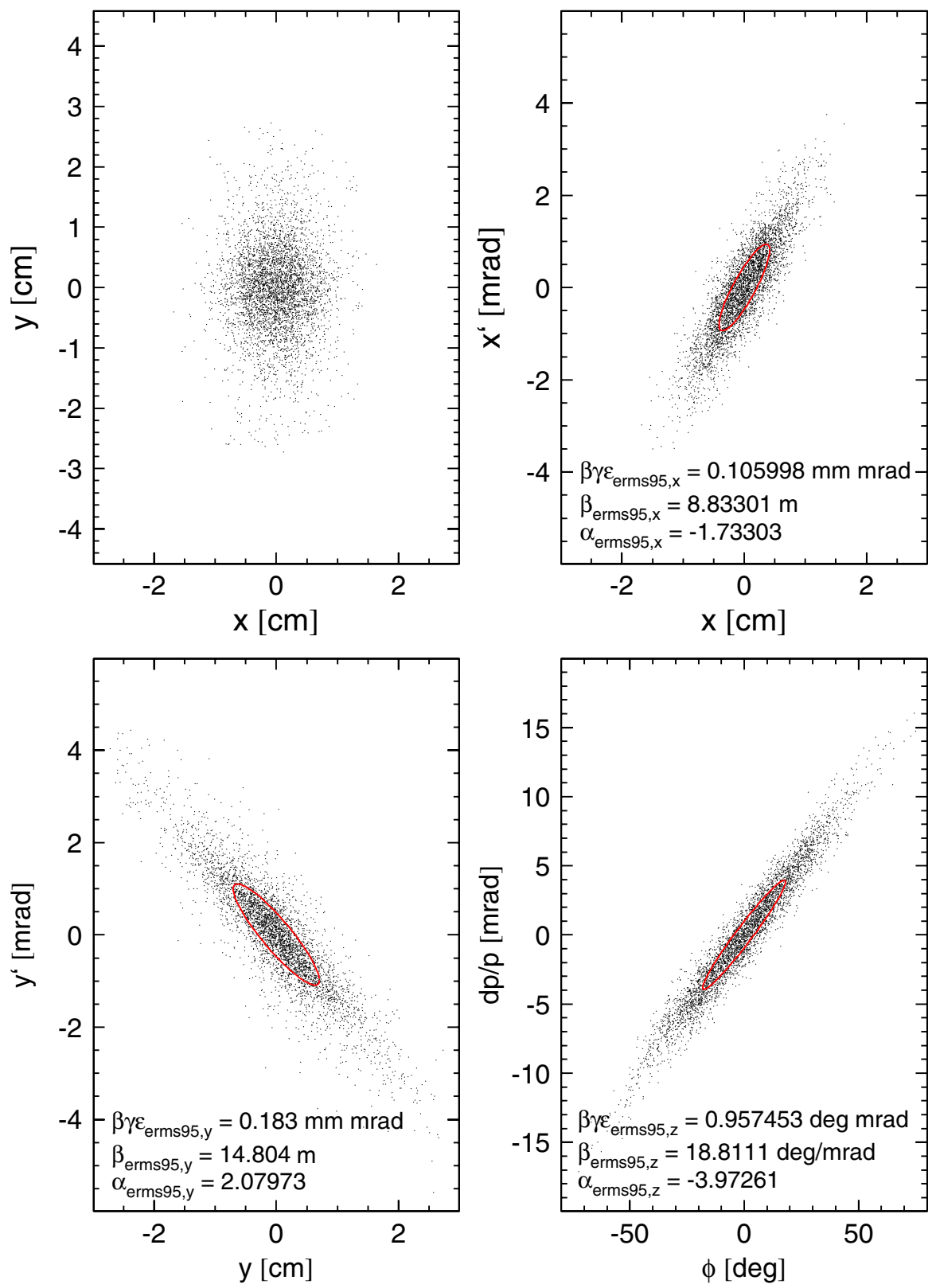

FIG. 9. (Color) Initial distribution used for the DYNAMION simulations (4300 particles). The 95\%-rms-Twiss parameters from $95 \%$ of the particles are indicated. The phase refers to $36 \mathrm{MHz}$. 
ones are obtained from the emittance measurements together with the application of the procedure defined in Sec. VA.

The distribution function $f$ must provide for reproduction of the brilliance curves in both transverse planes. In order to find such a function, many different expressions were tried. We found a function by defining a radius $\tilde{R}$ in the generalized six-dimensional phase space as

$$
\tilde{R}^{2}=X^{2}+X^{12}+Y^{1.2}+Y^{11.2}+\Phi^{2}+(\delta P / P)^{2}
$$

and

$$
\begin{gathered}
f(\tilde{R})=\frac{a}{2.5 \times 10^{-4}+\tilde{R}^{10}}, \quad \tilde{R} \leq 1 \\
f(\tilde{R})=0, \quad \tilde{R}>1,
\end{gathered}
$$

where $a$ is the normalization constant and the constant in the denominator results from the cutoff condition at $\tilde{R}=1$. By defining the radius $\tilde{R}$ using different powers for different sub-phase-spaces, the curvatures of the respective brilliance curves could be reproduced. Increasing the power to infinity for instance would deliver a homogeneous distribution in the respective sub-phase-space projection. Figures 7 and 8 plot the brilliance curves obtained from the distribution function $f$. The shape of the measured horizontal and vertical brilliance curve is reproduced very well, respectively.

Since for the longitudinal phase space distribution no measurement but on the rms bunch length is available, a Gaussian distribution cut at $4 \sigma$ is assumed. As seen from Fig. 7, this can be achieved by setting the respective powers in the definition of $\tilde{R}$ to a value of 2 .

The distribution was reconstructed from measurements delivering just a few of the possible projections of the sixdimensional distribution. Accordingly, there might be many functions $f^{*}$ that match the measured projections. However, concerning the ambiguity on longitudinal input, simulations were performed using different types of longitudinal distributions. The final transverse emittances did effectively not change with the longitudinal distribution type but they changed considerably with the transverse distribution type. For instance, in [15] results are presented that were obtained if a Gaussian cut at $2 \sigma$ is used in all three planes.

The distribution reconstructed from $f$ (Fig. 9) was used for simulations using DYNAMION (4300 particles), PARMILA, and PARTRAN. The last two codes used identical distributions of $2 \times 10^{5}$ particles in order to assure convergence of the results with respect to the number of particles. In the case of DYNAMION convergence was observed as well but the computing time of about 20 hours exceeds the computing times of the other codes by about 1.5 magnitudes.

\section{COMPARISON OF EXPERIMENTAL RESULTS WITH SIMULATION RESULTS}

For all zero current phase advances $\sigma_{o}$ ranging from $35^{\circ}$ to $90^{\circ}$ full beam transmission was observed through the DTL in the experiment. The simulation codes revealed losses of about $2 \%$. Generally, the transmissions in the simulations slightly drop with higher phase advances as plotted in Fig. 10. Including the measurements performed to check the reproducibility, in total 19 horizontal and vertical phase space distributions behind the DTL were recorded, respectively. Figures 11 and 12 show final phase space distributions at the DTL output as obtained from the measurements and from the simulations for three different values of $\sigma_{o}$.

For all $\sigma_{o}$ the simulations delivered final phase space distributions with elliptical symmetry. Just at highest phase advances two symmetric wings attached to the core are observed in the horizontal distributions. These wings were reproduced by all codes. The measured distributions for very low and very high $\sigma_{o}$ gave inhomogeneous shapes as seen for the $\sigma_{o}=35^{\circ}$ case at the left of Fig. 11. The simulated shapes look similar for all codes and did not reveal inhomogeneous shapes.

Longitudinal distributions at different locations along the DTL as obtained from DYNAMION simulations are displayed in Fig. 13. Their shapes depend weakly on the transverse phase advance. Filamentation occurs due to the large initial longitudinal emittance.

Figures 14-16 depict 100\% rms emittances along the beam line as obtained from the simulations. Since the rms emittances are extracted from $100 \%$ of the particles, the curves show spikes driven by very few particles with high amplitudes. However, the figures display the qualitative behavior of the emittances along the beam line. For all phase advances, the codes reveal that the major part of the overall emittance growth occurs at the beginning of the DTL. Along DTL tanks A3 and A4, the growth reached

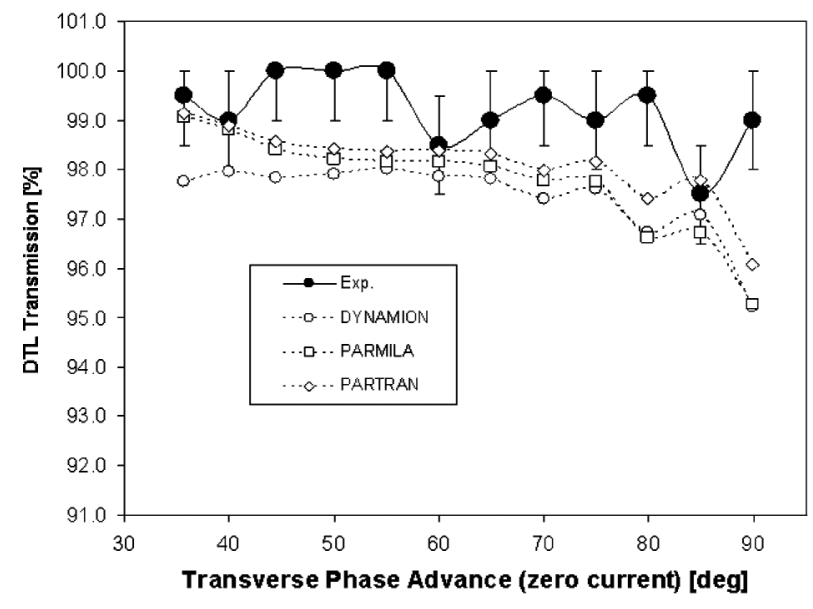

FIG. 10. Beam transmission through the DTL as a function of the transverse zero current phase advance $\sigma_{o}$ as measured and as simulated by the different codes. 


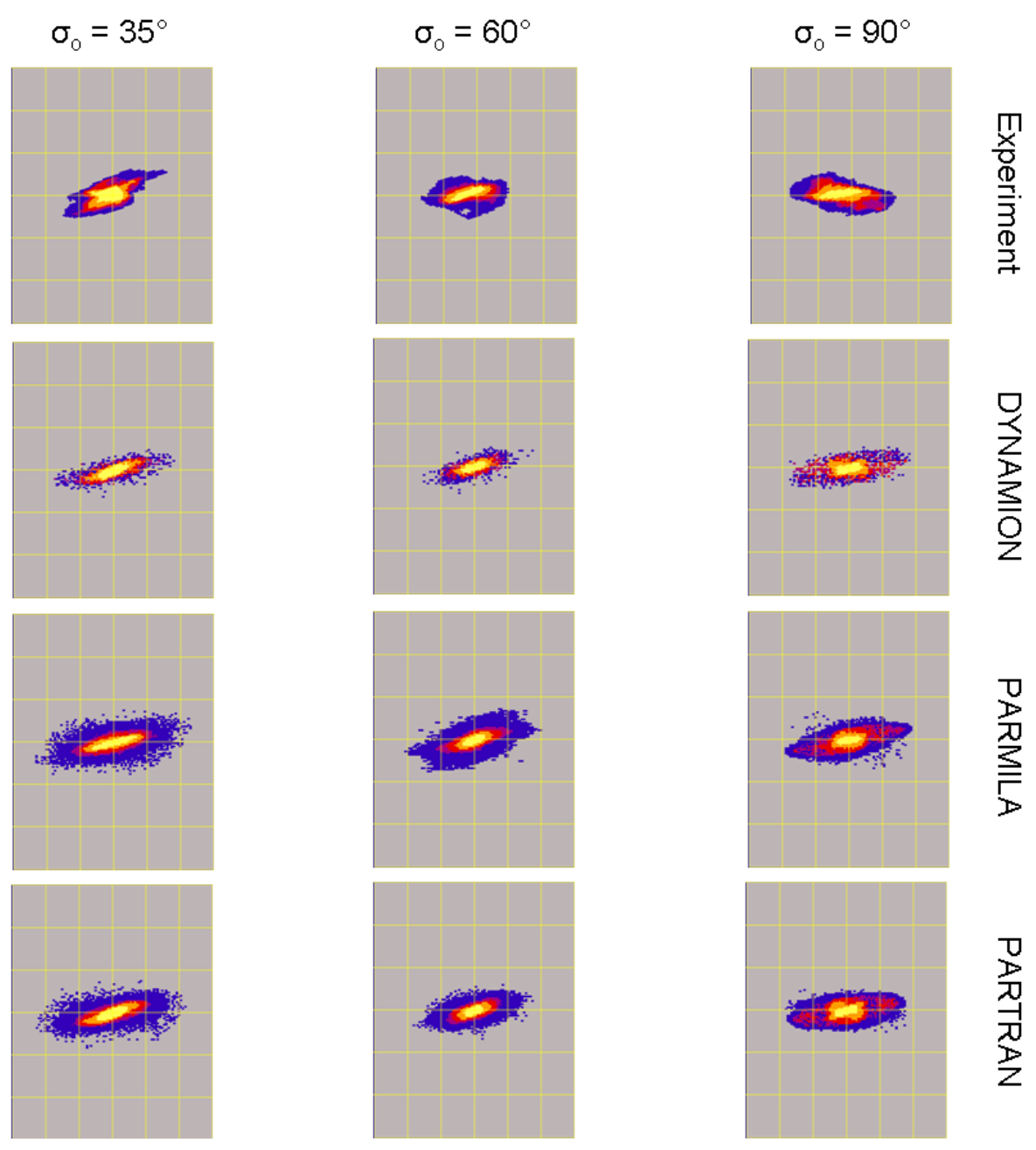

FIG. 11. (Color) From top to bottom: horizontal phase space distributions as measured and simulated at the exit of the DTL using DYNAMION, PARMILA, and PARTRAN. Left: $\sigma_{o}=35^{\circ}$; center: $\sigma_{o}=60^{\circ}$; right: $\sigma_{o}=90^{\circ}$. The scaling is $\pm 24 \mathrm{~mm}$ (horizontal axis) and $\pm 24 \mathrm{mrad}$ (vertical axis), respectively.

saturation (except for the horizontal plane at $\sigma_{o}=90^{\circ}$ ). These predictions from simulations are in very good agreement to previous measurements and simulations performed at the UNILAC at a phase advance of $53^{\circ}$ [15]. Growth is observed already along the section in front of the DTL where bunch compression in all three dimensions is applied in order to match the beam from the $36 \mathrm{MHz}$ section to the $108 \mathrm{MHz}$ DTL. At lowest and highest phase advances, the transverse emittances show a strong increase along the first cells of the first tank. The increase is driven by longitudinal rf-bucket overflow and by residual transverse mismatch as to be addressed below. PARMILA and PARTRAN produce very similar curves. Good agreement between these two codes has been already reported in [16]. The authors simulated the first DTL tank of the Spallation Neutron Source (SNS) using as input a distribution obtained from a previous RFQ simulation. The present study confirms that the good agreement also holds for a DTL with five tanks using initial distributions obtained from measurements.

The curve corresponding to DYNAMION is slightly above the previous ones. Because of the different treatment of longitudinal rf-bucket overflow within the codes, longitudinal emittances cannot be compared directly. The same applies to particle losses along the beam line.

From the experimental and the simulated data, the transverse $95 \% \mathrm{rms}$ emittances at the exit of the DTL were extracted as described in Secs. III and IV. The results are plotted in Figs. 17-19 as a function of the zero current phase advance $\sigma_{o}$ along the DTL. The horizontal emittances are plotted in Fig. 17, the vertical ones in Fig. 18, and the mean of the horizontal and the vertical emittance is plotted in Fig. 19. Generally lowest growth rates were found for phase advances close to $60^{\circ}$ experimentally and in simulations. However, scattering of the individual values around this general behavior is found. 

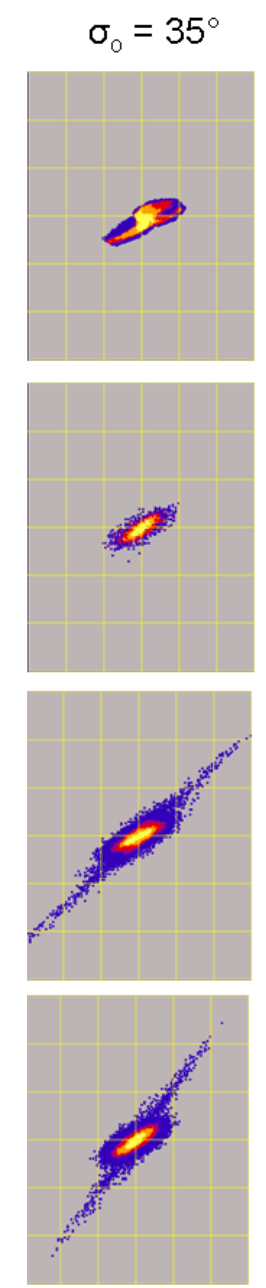

$\sigma_{0}=60^{\circ}$
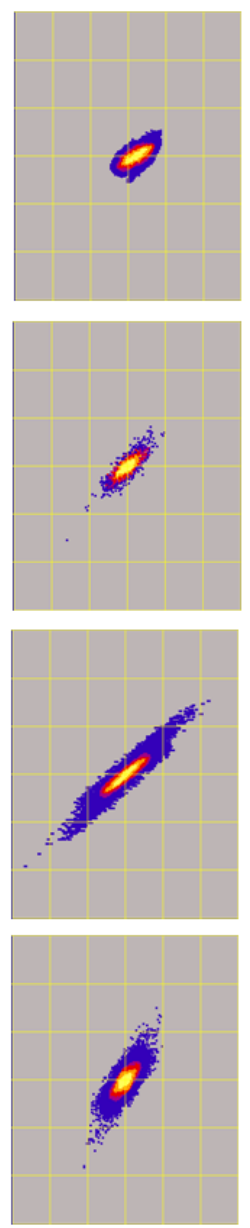

$\sigma_{0}=90^{\circ}$
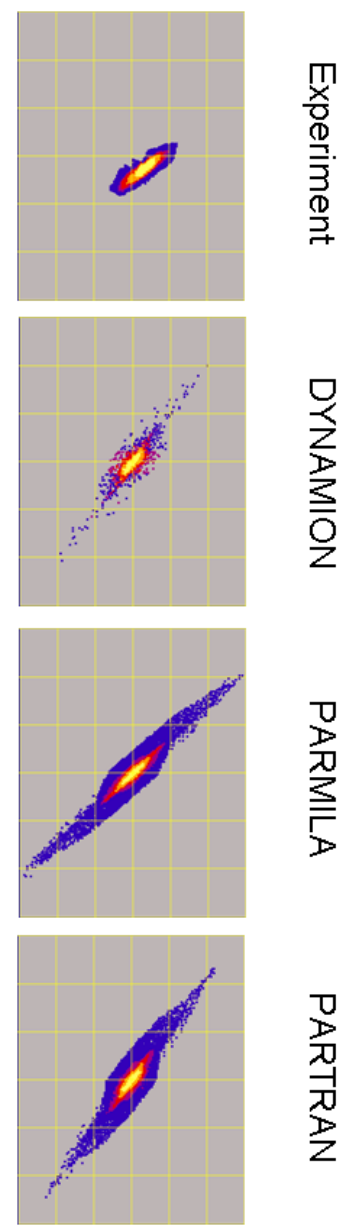

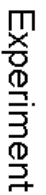

$\frac{\varrho}{3}$

FIG. 12. (Color) From top to bottom: vertical phase space distributions as measured and simulated at the exit of the DTL using DYNAMION, PARMILA, and PARTRAN. Left: $\sigma_{o}=35^{\circ}$; center: $\sigma_{o}=60^{\circ}$; right: $\sigma_{o}=90^{\circ}$. The scaling is \pm 36 mm (horizontal axis) and $\pm 30 \mathrm{mrad}$ (vertical axis), respectively.

The codes predict very similar dependence of the horizontal and vertical growth on $\sigma_{o}$, i.e., a minimum at intermediate phase advances and significant growth for very low and very high phase advances. This behavior is in agreement with the measurements.

In the vertical plane (Fig. 18) high growth rates were measured for the lowest phase advances of $35^{\circ}$ and $40^{\circ}$ exceeding the rates measured horizontally. This behavior was not reproduced by any of the simulation codes. The codes rather predict weak dependence on the phase advance for $\sigma_{o} \leq 60^{\circ}$. For higher phase advances, a slight increase of the growth was measured while the codes predict stronger growth. As already observed in the horizontal plane the codes produce similar dependence of the vertical emittance growth on $\sigma_{o}$. The resulting rms emittances of the codes differ just slightly from each other. For $\sigma_{o} \geq 50^{\circ}$ the emittances from the codes agree with the measurements within the error bars. For high phase advances the difference among the codes are largest.
Evaluating the mean values of horizontal and vertical emittances (Fig. 19) results in a smoother behavior with respect to the behavior in the single planes. In the measurements a broad minimum of the rms emittance growth is found at $\sigma_{o} \approx 60^{\circ}$. The scatter around this general behavior is reduced with respect to the scatter observed in the single planes. A possible reason for that might be emittance exchange between the two transverse planes. The driving terms could be nonlinear lattice components that were not included into the simulations as for instance quadrupole fringe fields and slight rotations of the quadrupoles around the design beam axis. For the mean growth, the codes predict a weak dependence on $\sigma_{o}$ at low phase advances. However, in agreement with the measurements lowest growth is found at about $60^{\circ}$. The codes reproduce the measured values within the error bars.

All codes predict a dominant peak of the (especially vertical) emittance at $70^{\circ}$, although the height of the peak depends on the code. This significant (vertical) peak at $70^{\circ}$ 


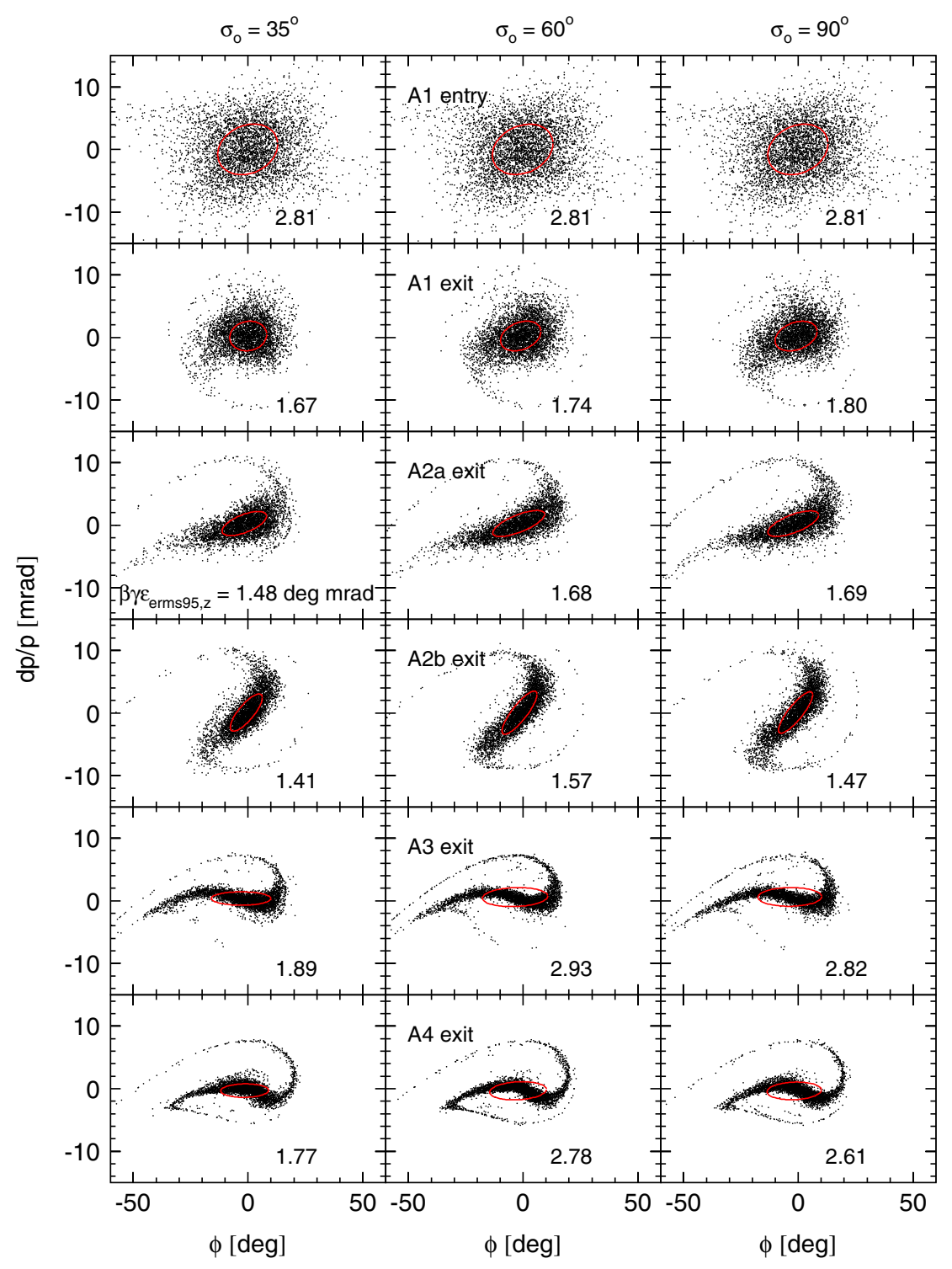

FIG. 13. (Color) DYNAMION-simulated longitudinal phase space distributions at the end of the tanks of the DTL for transverse zero current phase advances $\sigma_{o}$ of $35^{\circ}$ (left), $60^{\circ}$ (center), and $90^{\circ}$ (right). The specific tank and the corresponding normalized longitudinal $95 \% \mathrm{rms}$ emittance are indicated. The emittance refers to $108 \mathrm{MHz}$ and to the relative momentum deviation.

was not seen in the experiment. Instead the measurements revealed a vertical peak at $75^{\circ}$ although its strength is less with respect to the one observed in simulations at $70^{\circ}$. For the time being, no convincing explanation for these peaks can be given.

It might be argued if the observed differences of measured and simulated emittances is due to a wrong assumption on the initial longitudinal emittance. Simulations have been performed using initial longitudinal emittances of 2.3, 12 , and $37 \mathrm{deg} \operatorname{mrad}(36 \mathrm{MHz})$ for the $45^{\circ}$ case. The final transverse rms emittances differed by $5 \%$ at maximum. This observed independence from the initial longitudinal emittance seems reasonable since the initial bunch length does not effectively change as long as the rules for phase space reconstructions motivated in Sec. VA are applied. It is the initial bunch length that enters into the space charge forces.

It must be mentioned that the simulations assume a machine without any errors. Such errors generally drive additional emittance growth [17]. The relative contribution of machine errors to the external fields is larger at low phase advances and decreases for higher phase advances. This is in good agreement to the observation that the codes give slightly smaller final emittances at low $\sigma_{o}$ with respect to the measurements and that the agreement improves at higher $\sigma_{o}$.

The amount of mismatch for each $\sigma_{o}$ was estimated using DYNAMION simulations by evaluating the simulated 


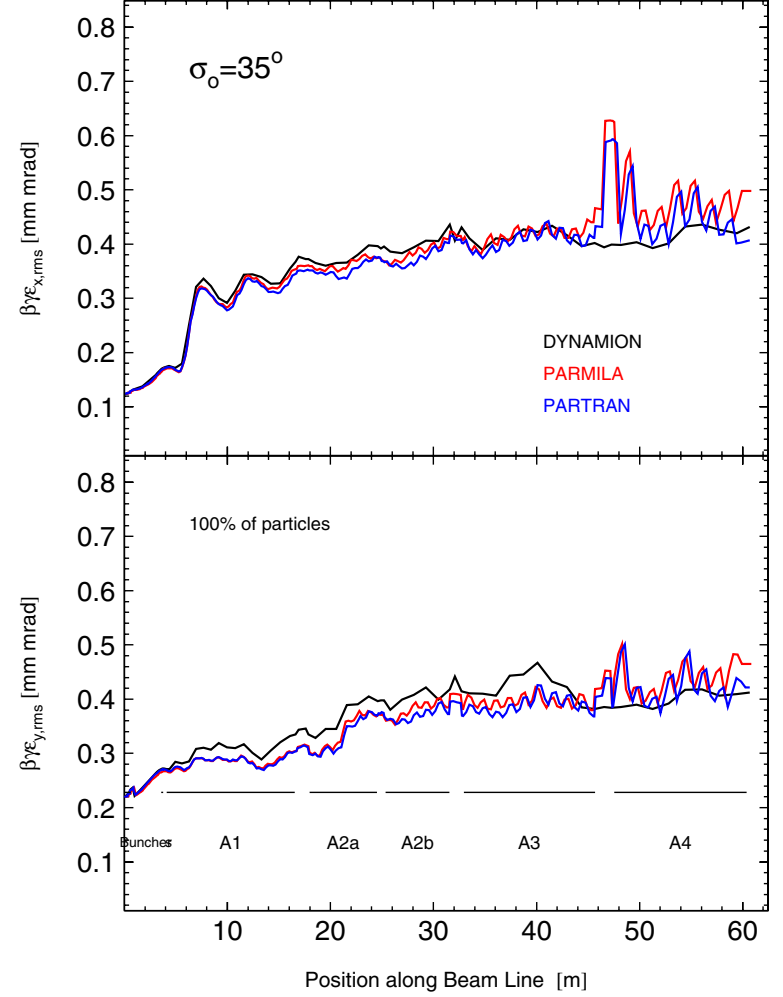

FIG. 14. (Color) Transverse normalized $100 \%$ rms emittances along the beam line as simulated by the three codes for a transverse zero current phase advance $\sigma_{o}$ of $35^{\circ}$.

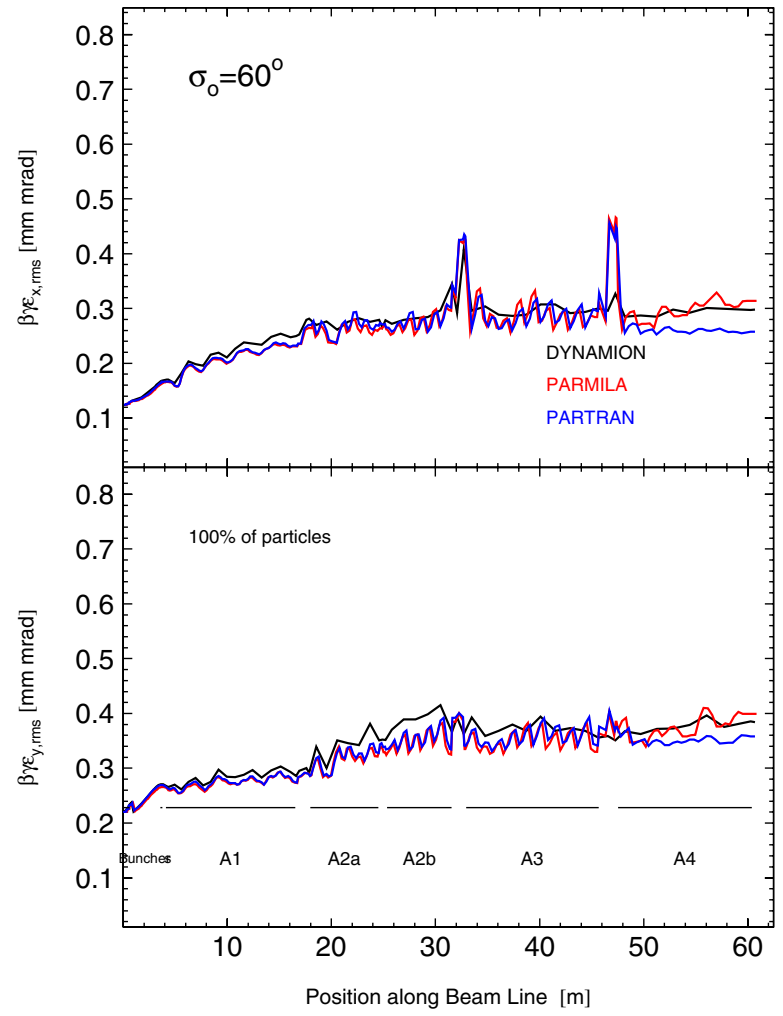

FIG. 15. (Color) Transverse normalized $100 \%$ rms emittances along the beam line as simulated by the three codes for a transverse zero current phase advance $\sigma_{o}$ of $60^{\circ}$.

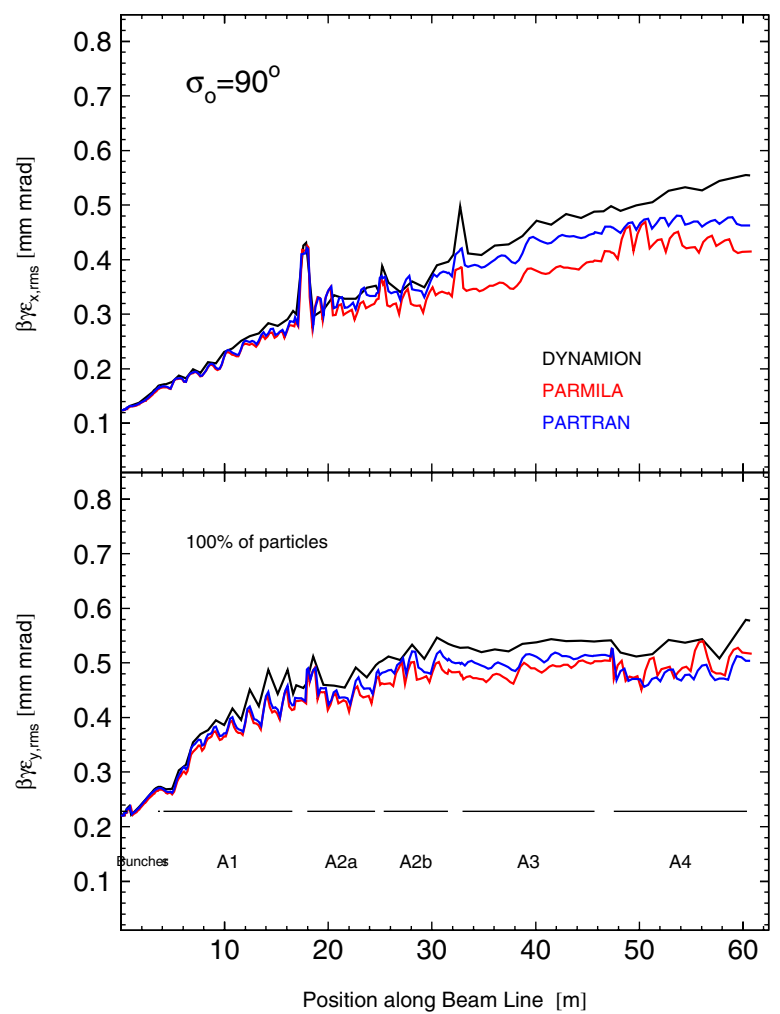

FIG. 16. (Color) Transverse normalized $100 \%$ rms emittances along the beam line as simulated by the three codes for a transverse zero current phase advance $\sigma_{o}$ of $90^{\circ}$.

phase space distributions at the entrance to the DTL and comparing their rms-Twiss parameters with the periodic DTL solutions. Figure 20 depicts phase space distributions right in front of the DTL. The ellipses corresponding to beam rms-Twiss parameters and to the periodic solutions of the DTL are drawn as well. From the two sets of Twiss parameters, the resulting mismatch parameter $M$ is calculated using the definition given in [18]:

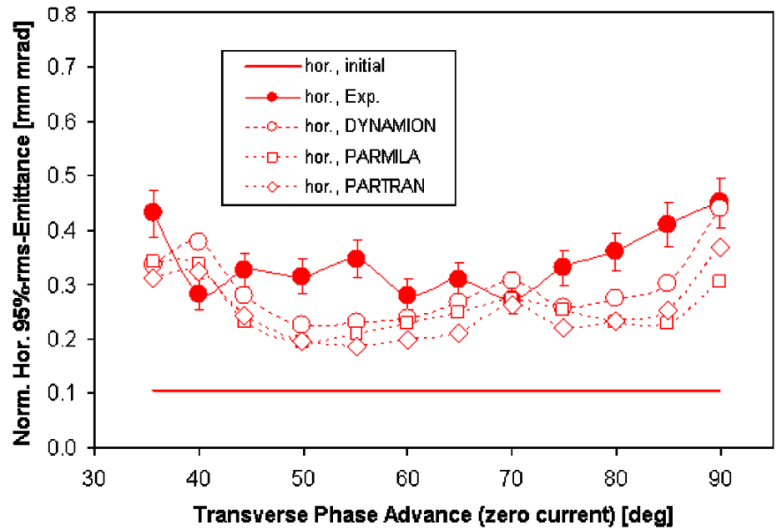

FIG. 17. (Color) Horizontal 95\% rms emittance at the end of the DTL as a function of the transverse zero current phase advance $\sigma_{o}$. 


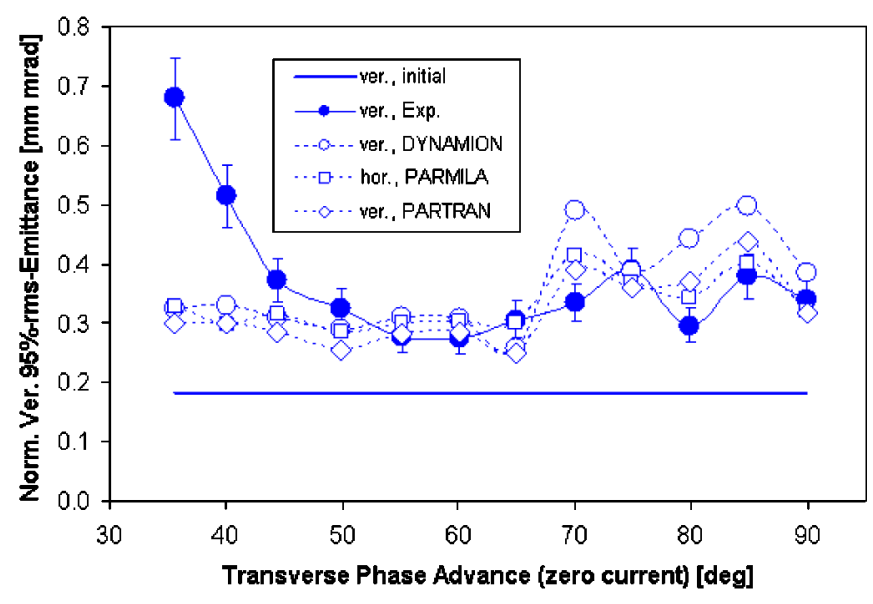

FIG. 18. (Color) Vertical $95 \% \mathrm{rms}$ emittance at the end of the DTL as a function of the transverse zero current phase advance $\sigma_{o}$.

$$
\begin{gathered}
M=\left[1+\frac{\Delta+\sqrt{\Delta(\Delta+4)}}{2}\right]^{1 / 2}-1 \\
\Delta=(\Delta \alpha)^{2}-\Delta \beta \Delta \gamma,
\end{gathered}
$$

where $\Delta \alpha=\left(\alpha_{\text {Ain }}-\alpha_{\text {per }}\right)$ is the difference between the alpha parameter of the beam at the entrance to the DTL and the alpha parameter of the periodic solution of the DTL including the defocusing space charge forces. $\Delta \beta$ and $\Delta \gamma$ are defined accordingly with $\gamma=\left(1+\alpha^{2}\right) / \beta$.

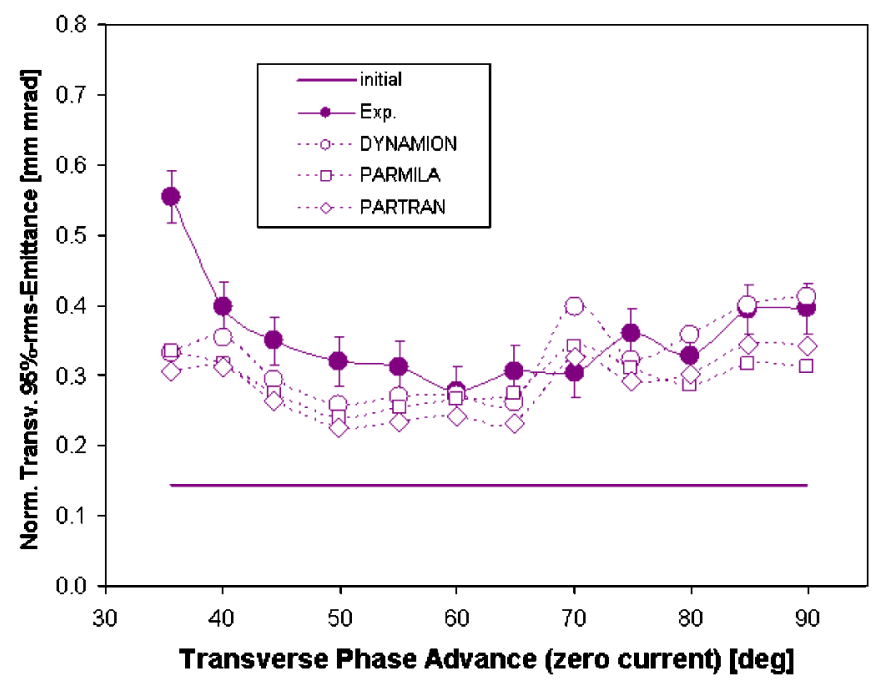

FIG. 19. (Color) Mean value of horizontal and vertical $95 \% \mathrm{rms}$ emittance at the end of the DTL as a function of the transverse zero current phase advance $\sigma_{o}$.

The mismatch parameters in the three planes were calculated for all phase advances applied during the experiment. They are plotted as a function of $\sigma_{o}$ in Fig. 21. It turned out that the transverse mismatch varied with the phase advance, while the longitudinal one remained constant. The low longitudinal mismatch for all values of $\sigma_{o}$ confirms the sensitivity of the machine setting with respect to correct longitudinal injection into the DTL. Large longitudinal mismatches would have been detected immedi-
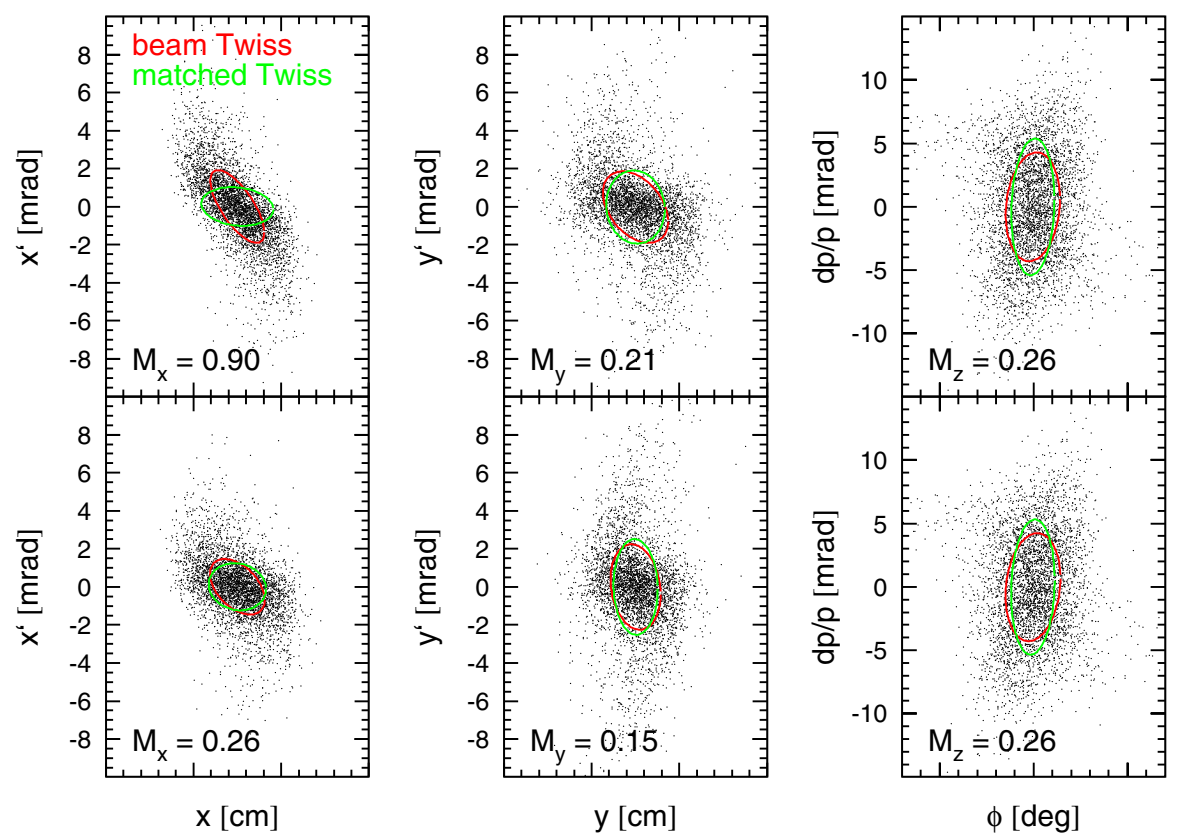

FIG. 20. (Color) Phase space distributions from DYNAMION simulations at the entrance to the DTL for different zero current phase advances $\sigma_{o}$ (upper: $35^{\circ}$; lower: $50^{\circ}$ ). The ellipses correspond to $95 \%$-rms-Twiss parameters of the distributions (red) and to the rmsmatched injection into the DTL (green). Phases refer to $108 \mathrm{MHz}$. 


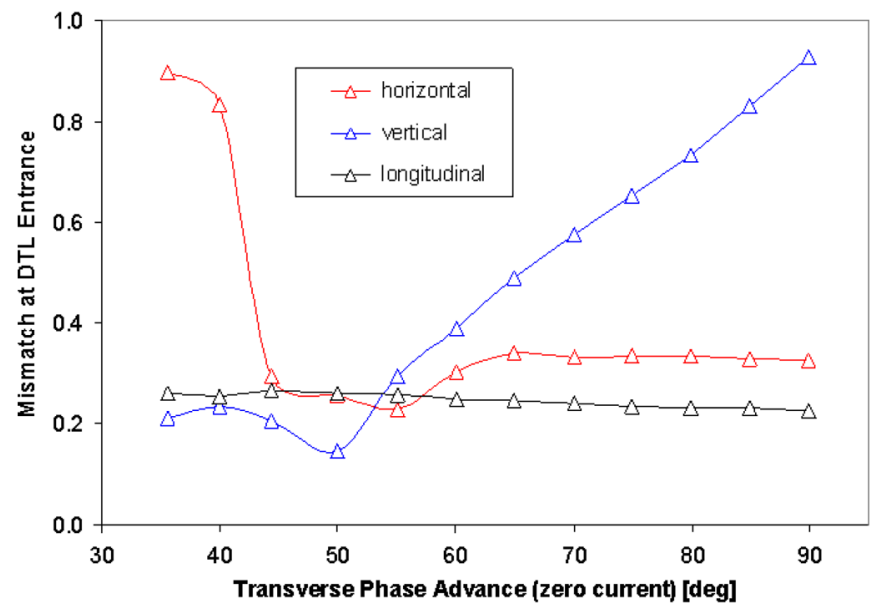

FIG. 21. (Color) Mismatch between beam 95\%-rms-Twiss parameters and Twiss parameters for rms-matched injection into the DTL as a function of the current phase advance $\sigma_{o}$.

ately during the experiment by the occurrence of transmission losses. This observation, together with the fact that the longitudinal mismatch is controlled by the two bunchers preceding the DTL, underlines the relevance of including these buncher settings into the method to reconstruct the initial phase space distribution for the simulations (see Sec. VA).

\section{SUMMARY AND CONCLUSION}

Measurements and simulations on transverse rmsemittance growth along an Alvarez DTL were performed. The campaign aimed at the study of the final DTL rms emittance as a function of the transverse focusing strength along the DTL. Measurements were compared with DYNAMION simulations using 4300 particles (3D-particleparticle interaction) and with PARMILA (V 2.32) and PARTRAN simulations using $2 \times 10^{5}$ particles (PICNIC3D solver). Special care was taken for the reconstruction of the initial distribution for the simulations. It was succeeded to obtain a detailed modeling beyond rms equivalence. The rms-Twiss parameters of the initial distribution were extracted from beam emittance measurements in front of the DTL. The type of distribution was obtained from fitting rms-brilliance curves to measurements, i.e., the fractional rms emittances as a function of the fraction.

Smallest DTL exit emittances were found at zero current phase advances of about $60^{\circ}$ experimentally and in simulations. The benchmarking revealed good agreement on average transverse emittances among the codes and the experiment. Just for small phase advances the measured emittances exceeded significantly the simulated ones.

We intend to extend the scope of the benchmarking, which so far focused on rms properties of the output, towards the formation of tails in the output distributions. Future efforts aim at the upgrade of longitudinal diagnos- tics in front of the DTL from rms bunch lengths to full phase space distributions. This will allow for measurements with further improved matching to the DTL.

\section{ACKNOWLEDGMENTS}

We acknowledge the support of the European Community-Research Infrastructure Activity under the FP6 "Structuring the European Research Area" program (CARE, Contract No. RII3-CT-2003-506395). The participation of D. Jeon to this work was made possible partly by the support of ORNL/SNS (managed by UT-Battelle, LLC, for the U.S. Department of Energy under Contract No. DEAC05-00OR22725).

\section{APPENDIX: REDUCTION OF MISMATCH AND OF EMITTANCE GROWTH}

The preparation of the simulations employed the procedure to reconstruct the Twiss parameters of the initial distribution as described in Sec. VA. Applying this procedure and using the reconstructed initial distributions as input for a dedicated rms-matching routine with space charge [19] is expected to lead to improved matching conditions. Experimental verification of reduced emittance growth by systematic rms matching would demonstrate that the applied method of reconstruction is valid.

Ten months after the benchmarking campaign one day of beam time was available to test the procedure experimentally. Although the amount of data that could be taken was small compared with the one of the benchmarking campaign, the main results shall be briefly reported.

$\mathrm{An}^{40} \mathrm{Ar}^{10+}$ beam of $7.1 \mathrm{~mA}$ was available to remeasure transverse emittances in front of and behind the DTL for zero current phase advances of $35^{\circ}, 60^{\circ}$, and $90^{\circ}$. The normalized $100 \%$ rms emittances in front of the DTL were measured as 0.14 (horizontally) and $0.28 \mathrm{~mm}$ mrad (vertically). The rms bunch length in front of the DTL was measured as well. A value of $19 \mathrm{deg}$ was found $(36 \mathrm{MHz})$. These values are comparable with those of the previous campaign $(0.12 / 0.23 \mathrm{~mm}$ mrad, $20 \mathrm{deg})$.

The rms matching including space charge forces was done based on the reconstructed initial distribution. The matching section was set for rms-matched injection into the DTL for each of the applied phase advances. The setting of the section in front of the DTL as proposed by the matching routine was applied including the two bunchers. Full beam transmission was obtained immediately.

Using the DYNAMION code the remaining mismatch at the injection into the DTL was estimated in an analogue way as described in Sec. VI. The mismatch was reduced significantly as demonstrated by comparing Fig. 22 with Fig. 21.

During the measurements described in this section, two neighboring quadrupole lenses in tank A3 of the DTL had to be switched off due to cooling water leakage. In order to 


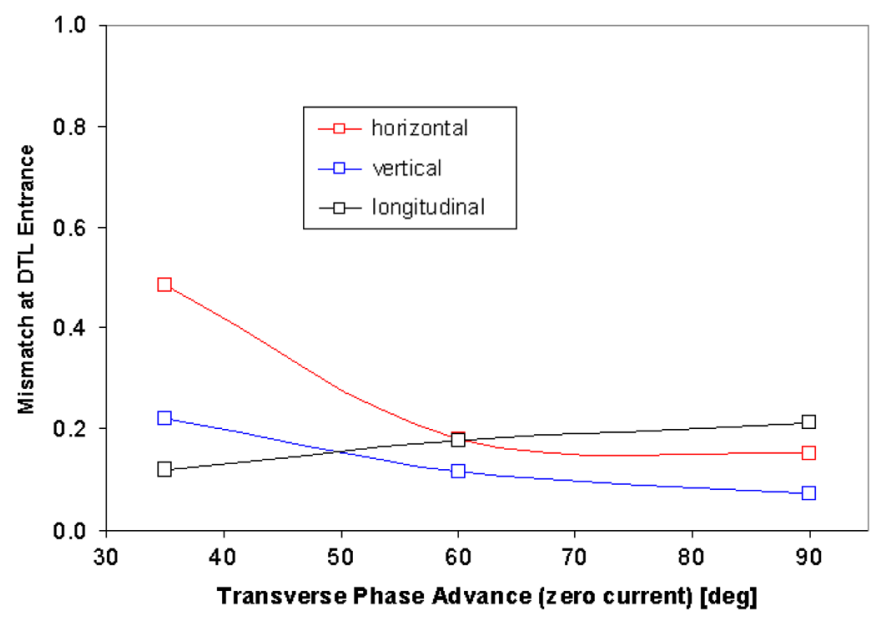

FIG. 22. (Color) Reduced mismatch between beam 95\%-rmsTwiss parameters and the Twiss parameters for the rms-matched injection into the DTL as a function of the current phase advance $\sigma_{o}$.

avoid mismatch in connection with longitudinal focusing, the rf power in tanks A3 and A4 was switched off. Transverse rms emittances were measured after tank A4. The emittance growth along the DTL tanks that are not rf powered (A3, A4) can be neglected with respect to the growth along rf-powered DTL tanks. This assumption was verified in simulations and experimentally as reported in detail in [15].

The rms emittances measured behind the DTL were normalized to the rms emittances measured in front of the DTL to extract the rms-emittance growth rates. The extracted growth rates are plotted in Fig. 23 together with the corresponding values of the benchmarking campaign. It could be demonstrated that the improved matching significantly reduced the transverse rms-emittance growth.

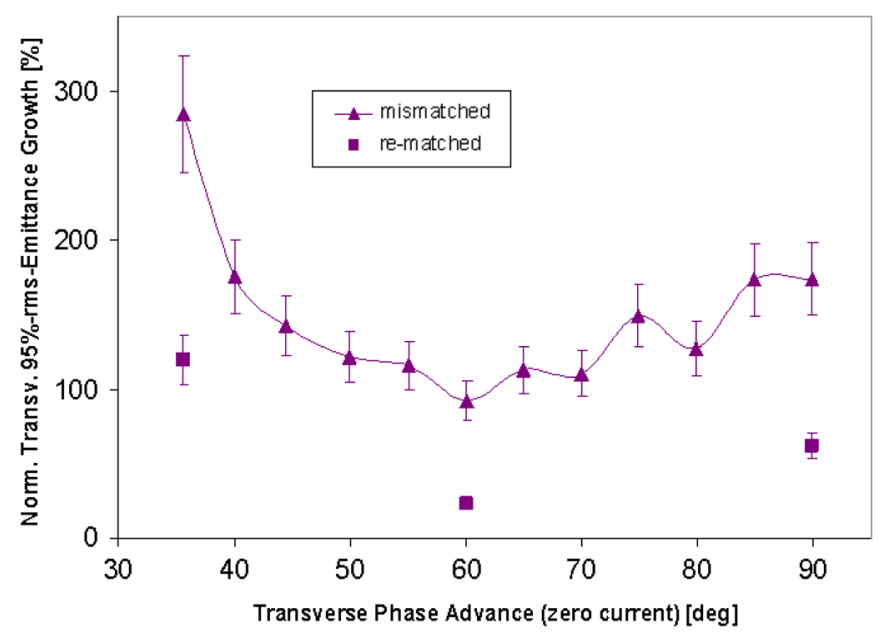

FIG. 23. (Color) Growth of the mean value of horizontal and vertical $95 \%$ rms emittance along the DTL as a function of the zero current phase advance $\sigma_{o}$.
Especially the mismatches for the $35^{\circ}$ and the $90^{\circ}$ cases were reduced leading to lower DTL exit emittances. The growth for the $60^{\circ}$ case (being relevant for production runs of the UNILAC) was reduced from $90 \%$ to $20 \%$ being an essential gain for machine operation. The measurements confirmed that a valid procedure was applied during the benchmarking to reconstruct initial Twiss parameters in front of the DTL.

[1] "FAIR Baseline Technical Report," Vol. 2, GSI Darmstadt, Germany, 2006, p. 335.

[2] W. Barth, L. Dahl, L. Groening, J. Glatz, S. Richter, and S. Yaramyshev, in Proceedings of the XXII Linac Conference, Lübeck, Germany, 2004, p. 246.

[3] http://care.lal.in2p3.fr/.

[4] S. Yaramyshev, W. Barth, L. Groening, A. Kolomiets, and T. Tretyakova, Nucl. Instrum. Methods Phys. Res., Sect. A 558, 90 (2006).

[5] J.H. Billen and H. Takeda, PARMILA Manual, Report No. LAUR-98-4478, Los Alamos, 1998 (Revised 2004).

[6] R. Duperrier, N. Pichoff, and D. Uriot, ICSS 2002 Conference, Amsterdam, The Netherlands, 2002.

[7] C. K. Allen, K. C. D. Chan, P. L. Colestock, K. R. Crandall, R. W. Gernett, J. D. Gilpatrick, W. Lysenko, J. Qiang, J. D. Schneider, M. E. Schulze, R. L. Sheffield, H. V. Smith, and T. P. Wangler, Phys. Rev. Lett. 89, 214802 (2002).

[8] P. L. Colestock, D. Gilpatrick, M. E. Schulze, H. V. Smith, and T. P. Wangler, Phys. Rev. ST Accel. Beams 5, 124201 (2002).

[9] A. Franchi, W. Bayer, G. Franchetti, L. Groening, I. Hofmann, A. Orzhekhovskaya, S. Yaramyshev, X. Yin, A. Sauer, R. Tiede, G. Clemente, R. Dupperrier, D. Uriot, G. Bellodi, F. Gerigk, A. Lombardi, and T. Mütze, in Proceedings of the XXIII Linac Conference, Knoxville, TN, 2006, p. 462.

[10] A. Franchi, W. Bayer, G. Franchetti, L. Groening, I. Hofmann, A. Orzhekhovskaya, S. Yaramyshev, X. Yin, A. Sauer, R. Tiede, G. Clemente, R. Dupperrier, D. Uriot, G. Bellodi, F. Gerigk, A. Lombardi, and T. Mütze, http:// www-dapnia.cea.fr/Phocea/file.php?class $=$ std $\& \&$ file $=$ Doc/Care/note-2006-011-HIPPI.pdf.

[11] L. Groening and W. Barth, in Proceedings of the 21st International Linac Conference, Gyeongju, Korea, 2002 (Pohang Accelerator Laboratory, Pohang, Korea, 2002).

[12] P. Forck, F. Heymach, T. Hoffmann, A. Peters, and P. Strehl, in Proceedings of the 20th International Linac Conference, Monterey, CA, 2000 (SLAC, Menlo Park, CA, 2000).

[13] G. Riehl, PROEMI: An emittance measurement and evaluation code. The manual is available from the code author on request (g.riehl@gsi.de).

[14] T. P. Wangler, Rf Linear Accelerators (John Wiley \& Sons Inc., New York, 1998), p. 278.

[15] L. Groening, W. Barth, W. Bayer, L. Dahl, P. Forck, P. Gerhard, I. Hofmann, G. Riehl, S. Yaramyshev, and D. Jeon, http://www-dapnia.cea.fr/Phocea/file.php?class= std\&\&file=Doc/Care/care-report-07-030.pdf. 
[16] S. Nath, J. Qiang, R. Ryne, J. Stovall, H. Takeda, L. Young, K. R. Crandall, N. Pichoff, and D. Uriot, in Proceedings of the Particle Accelerator Conference, Chicago, IL, 2001 (IEEE, New York, 2001).

[17] K. R. Crandall, AOT Division Technical Report LA-CP-
96-16, 1966.

[18] See Ref. [14] p. 217.

[19] L. Groening, http://www-linux.gsi.de/ lgroenin/bd_ unilac/HSI/Alvarez_Matching.html. 